\title{
Las bases curriculares 2013: Ios desafíos actuales para la formación inicial y aprendizaje histórico de profesores de historia en Chile
}

\section{As bases curriculares 2013: os desafios atuais para a formação inicial e aprendizagem histórica de professores de história no Chile}

\section{The curricular bases 2013: current challenges for initial training and historical learning of history teachers in Chile}

\author{
Gabriela Vásquez Leyton ${ }^{1}$
}

\begin{abstract}
RESUMEN
El artículo que se presenta tiene por finalidad revisar las bases curriculares 2013 propuestas para la asignatura de historia, geografía y ciencias sociales de $7^{\circ}$ básico a $2^{\circ}$ medio, con el objetivo de definir los retos para la formación inicial de profesores de historia en Chile y, por ende, estipular posibles estrategias de enseñanza tanto para los formadores de profesores como para los investigadores en didáctica de la historia y de las ciencias sociales. Estos desafíos se relacionan, principalmente, con incluir en el proceso de aprender a enseñar el desarrollo del pensamiento histórico y el desarrollo de las habilidades cognitivas que permitan la comprensión profunda de los procesos históricos tales como pensamiento temporal y espacial, análisis y trabajo con fuentes, pensamiento crítico y comunicación.

Palabras-clave: pensamiento histórico; currículum escolar; formación de profesores; didáctica de la historia.

DOI: $10.1590 / 0104-4060.46005$

1 Pontificia Universidad Católica de Valparaíso. Valparaíso, Chile. Avenida Brasil, nº 2950.
\end{abstract} E-mail: gabriela.vasquez@pucv.cl 


\title{
RESUMO
}

O presente artigo tem por objetivo revisar as bases curriculares de 2013 propostas para a disciplina de história, geografia e ciências sociais do $7^{\circ}$ ano da Educação Básica ao $2^{\circ}$ ano do Ensino Médio, com o objetivo de definir os desafios para a formação inicial de professores de história no Chile e, portanto, estipular possíveis estratégias de ensino tanto para os formadores de professores como para os pesquisadores da didática da história e das ciências sociais. Estes desafios dizem respeito a incluir no processo de aprender a ensinar o desenvolvimento do pensamento histórico e o desenvolvimento de habilidades cognitivas que permitem profunda compreensão dos processos históricos, tais como o pensamento temporal e espacial, a análise e o trabalho com fontes, o pensamento crítico e a comunicação.

Palavras-chave: pensamento histórico; currículo escolar; formação de professores; didática da história.

\begin{abstract}
The purpose of the present article is to review the 2013 curriculum proposed for history, geography and social sciences, from the $7^{\text {th }}$ grade of Primary School to the $2^{\text {nd }}$ grade of Secondary School, in order to define challenges for the initial history teachers' training programmes in Chile and, therefore, to establish teaching strategies for teacher trainers and researchers in didactics on history and social sciences education. Those challenges intend to include in the learning how to teach process the developing of historical thinking and cognitive skills that allow deep comprehension of the historical processes such as temporal and spatial thinking, the work and studies on sources, critical thinking and communication.
\end{abstract}

Keywords: historical thinking; school curriculum; professor training; teaching of history.

\section{Introducción: bases curriculares}

"Una formación que consideramos una necesidad social puesto que las personas que carecen de ella se ven privadas de conocimientos útiles para el ejercicio de la ciudadanía."

(LOPEZ FACAL, 2014, p. 273) 
La asignatura de historia, geografía y ciencias sociales se considera que tiene implícita distintas funcionalidades que buscan desarrollar en el estudiante competencias que le permitan en su vida adulta enfrentar los desafíos ciudadanos y desenvolverse activamente en el sistema democrático actual. Al respecto, hay autores que enfatizan la necesidad social de ampliar el pensamiento racional y objetivo de aquellos sucesos que ocurren en el presente y ocurrieron en el pasado; con la idea de potenciar la conciencia de los ciudadanos y, de esta manera, hacerlos conscientes a la hora de valorar, analizar, participar y tratar de solucionar los problemas de la sociedad contemporánea que los envuelven y de los cuales son parte. (PRATS, 2011, p. 14).

Estos principios son parte de las denominadas "Bases Curriculares"2 que corresponden al nuevo marco que delinea el currículum escolar en Chile. En este sentido, la propuesta a revisar en estas páginas corresponde al primer ciclo de educación secundaria ( $7^{\circ}$ básico $-2^{\circ}$ medio), donde se propone continuar con el desarrollo de los aprendizajes alcanzados durante la etapa escolar anterior (educación primaria), considerando que un estudiante al egresar de $6^{\circ}$ básico ha adquirido un sentido de identidad y de pertenencia a nuestra sociedad. Además, es capaz de reconocer que la realidad social en la que se desenvuelve es compleja, desarrollando una visión comprensiva respecto de los contenidos históricos, geográficos y sociales estudiados. Así mismo, conoce sus derechos y sus deberes como ciudadano, reconoce el territorio como contexto de distintas actividades humanas y lo caracteriza integrando variables físicas y humanas. De esta forma, reconoce los distintos modos en que las personas se han organizado y resuelto problemas comunes a la humanidad fortaleciendo su perspectiva histórica. (MINEDUC, 2013, p. 194).

En concordancia con lo expuesto anteriormente, para delinear la progresión del aprendizaje durante el ciclo secundario las bases curriculares proponen desarrollar distintos énfasis tanto en lo histórico como en lo geográfico, además ponderan lo multidisciplinar considerando la perspectiva política y económica de las ciencias sociales así como su carácter interpretativo, entiendo que la construcción de conocimiento escolar no puede ser realizada de manera parcializada o fragmentada, sino más bien se convierte en significativa cuando utiliza metodologías de trabajo comunes que favorecen el aprendizaje activo y

2 Para el caso de educación básica (primaria) fueron elaboradas en el año 2012 y para los cursos de $7^{\circ}$ básico a $2^{\circ}$ medio en el año 2013. A su vez, los planes de estudio, las bases curriculares y los programas para $3^{\circ}$ y $4^{\circ}$ medio científico-humanista están en proceso de elaboración y se espera que estén actualizadas durante el presente año 2015. De esta forma, el Ministerio de Educación chileno implementará las nuevas bases de manera progresiva para el ciclo de $7^{\circ}$ a $2^{\circ}$ medio a partir del $2016,7^{\circ}$ y $8^{\circ}$ básico en el año 2016 ; las de $1^{\circ}$ medio en 2017 ; y las de $2^{\circ}$ medio en 2018 . Este cambio en el currículo se suma al implementado en 2013 para el primer ciclo de $1^{\circ}$ a $6^{\circ}$ básico. 
participativo. Junto con lo anterior, puntualizan en el desarrollo de competencias ciudadanas democráticas y respeto por los Derechos Humanos (DDHH), como parte del compromiso consciente de ser una sociedad que busca respaldar, valorar y consolidar la Democracia. Además, presenta la historia de Chile y del mundo de una manera complementaria e interrelacionada, como parte de un proceso común e interconectado, más que como una sucesión de hechos o acontecimientos independientes de la historia universal, sino más bien muchos de ellos se entienden considerando la perspectiva global, situación lo que se relaciona con el entendimiento de la multicausalidad histórica, que es una habilidad central dentro del pensamiento histórico y, por tanto, un objetivo a fortalecer en la enseñanza escolar.

De esta forma, específicamente, los énfasis de las bases curriculares se relacionan con:

- Perspectiva multidisciplinar;

- Pensamiento histórico;

- Pensamiento geográfico;

- Desarrollo de competencias ciudadanas y respeto por los derechos humanos;

- El carácter interpretativo de las ciencias sociales;

- La historia de Chile y del mundo bajo una mirada dialógica;

- Las ciencias sociales: perspectiva política y económica.

Estos principios se relacionan con el objetivo transversal de la asignatura que desde $1^{\circ}$ básico hasta $4^{\circ}$ medio plantea que el estudiante pueda alcanzar una mejor comprensión de su sociedad y de su rol en ella. Así mismo, para el ciclo en estudio ( $7^{\circ}$ básico a $2^{\circ}$ medio), concretamente, se establece que los estudiantes deben comprender la complejidad de la realidad social y el devenir de nuestra sociedad, estableciendo que nuestra disciplina debe convertirse en un aporte significativo para enfrentar los desafíos de desenvolverse en un mundo cada vez más dinámico, plural y cambiante. (MINEDUC, 2013, p. 194).

Estos principios siguen el mismo lineamiento considerado en el marco curricular anterior, donde se propone desarrollar en los estudiantes conocimientos, habilidades y disposiciones que les permitan estructurar una comprensión del entorno social y su devenir histórico, así como se les orienten a actuar crítica y responsablemente en la sociedad, sobre la base de los principios de solidaridad, pluralismo, cuidado del medio ambiente, valoración de la democracia y de la identidad nacional. (MINEDUC, 2009, p. 195).

Tal como podemos observar, desde el ámbito cognitivo este referente buscaba que los alumnos de educación secundaria incorporen a su formación académica las herramientas que son necesarias para enfrentar los desafíos que les impone su existencia en un mundo que cambia aceleradamente y que es 
cada vez más complejo e interconectado. (MINEDUC, 2005, p. 95). Al mismo tiempo, se pretendía que los alumnos perciban que la historia no constituye un saber desvinculado de su mundo (MINEDUC, 2009, p. 2) y de realidad más cercana, sino que esta asignatura les puede ayudar a desarrollar capacidades que les permitan asumir los retos, desafíos y posibilidades que presenta vivir y ser parte de una sociedad democrática.

En este sentido, estos aspectos que representan un continuismo curricular se consideran que están en concordancia con los principios de una formación democrática de la ciudadanía maximalista, principio central para la educación de un país que busca constantemente consolidar las instituciones y funcionalidades democráticas. Por ello, desde la didáctica disciplinar el fortalecimiento de estas competencias ciudadanas en el aula escolar se desarrollan, por ejemplo, trabajando argumentaciones o debates así como motivando a los alumnos a participar activamente en los procesos ciudadanos o presentándole distintas estrategias para la resolución de problemas de su propio entorno y comunidad socio-cultural. De esta manera, se orienta la enseñanza de la historia para el desarrollo del pensamiento y para la comprensión profunda de contenidos implicados, además, promoviendo la participación y discusión en la clase se permite a desplegar altos niveles de compromiso en su aprendizaje por parte de los alumnos. (EGGEN, 2001, p. 27). Por tanto, la formación inicial de los profesores debe considerar estas formas activas de aprendizaje en sus procesos formativos referidos al proceso de aprender a enseñar.

También el currículo escolar chileno considera que la asignatura se conforma por distintas disciplinas de las ciencias sociales, que estudian desde diversas perspectivas al ser humano en el tiempo y en el espacio, así como de manera individual y como colectiva, es decir, como miembro de la sociedad. Esta perspectiva otorga un enfoque multidisciplinario a la enseñanza y al aprendizaje, por ello se entiende que el trabajo conjunto de estas disciplinas permite al alumnos desarrollar conocimientos, habilidades y actitudes necesarias para comprender la compleja realidad social contemporánea y, junto a ello, su devenir histórico (MINEDUC, 2012, p. 78), adhiriendo su rol como ciudadano capaz de actuar en la sociedad y enfrentar los desafíos del mundo globalizado.

En este sentido, podemos observar que el currículo chileno considera los aprendizajes más que la adquisición de conceptos como el desarrollo de habilidades y actitudes asociadas desde una perspectiva global e integradora, en la que el conocimiento histórico y geográfico actuarían como ejes estructurantes de los procesos sociales pasados y recientes estudiados, a los cuales se les reconoce explicaciones multicausales a partir del uso de fuentes variadas y diversas. Por ello, se incluye como forma de trabajo la metodología de las ciencias sociales, con el fin de enseñar al estudiantado a buscar, organizar, sintetizar y comunicar 
información, permitiéndole ser capaz de llegar a explicaciones argumentadas y, de esta forma, establecer conclusiones propias y posibilitando la apertura al aprendizaje futuro de nuevos conocimientos sociales de manera progresiva pero también favoreciendo el aprendizaje autónomo, competencias que hacen posible tener las herramientas que permiten una actuación crítica y responsable en la sociedad.

\section{El desarrollo del pensamiento histórico}

El currículo escolar chileno contempla, para el ciclo $7^{\circ}$ básico a $2^{\circ}$ medio, que el estudiante alcance progresivamente una mejor comprensión de su presente, considerando que es esencial que comprenda su pasado y la relación que este tiene con su vida diaria, con su identidad y con su entorno (MINEDUC, 2013, p. 195), idea que fundamentaría el desarrollo del pensamiento histórico, en cuanto es necesario que comprendan que la experiencia de vivir en sociedad está contextualizada en el tiempo, ya que nuestra existencia se desenvuelve en el devenir de la historia. Por lo anterior, uno de los primeros elementos a considerar a la hora de diseñar e implementar las asignaturas que impliquen el aprender a enseñar hace referencia a considerar aquellos aspectos que permiten el fortalecimiento de estas competencias históricas en el mundo escolar, principalmente introducir el método histórico en las actividades y en los procedimientos de la sala de clases, centrándose en un conocimiento científico de la historia más que relatos no verificables sobre el pasado. De ahí la importancia de que a los jóvenes estudiantes se les inicie en el trabajo científico siguiendo las mismas pautas que utilizan los historiadores. (FELIÚ; HERNÁNDEZ, 2011, p. 48).

En este sentido, un estudio realizado con profesores en ejercicio del sistema escolar en el contexto nacional estableció que dentro de las competencias que debiese tener un profesor de Historia ${ }^{3} \mathrm{y}$, que por lo tanto, deben ser preponderante en los currículos de formación docente se consideran las competencias relacionadas con la construcción del pensamiento histórico tales como: pensamiento cronológico y espacial' la investigación, las evidencias y los puntos de vista y, por último, la interpretación histórica relacionada con la multicausalidad. (VÁSQUEZ; MONTECINOS, 2007, p. 27).

Así el pensar históricamente, podemos relacionarlo con la comprensión

3 Estas ideas forman parte de los resultados de investigación del proyecto DGI 183.7932005, financiado por la Pontificia Universidad Católica de Valparaíso, titulado: "Competencias disciplinarias del eje de práctica docente en la formación del profesor de historia, geografía y ciencias sociales". 
del conocimiento histórico y está referido a la acción de significar el pasado a través del uso deliberado de estrategias creadas por los historiadores como son el tiempo histórico, la causalidad, la objetividad, la intertextualidad, la cual se presenta en una forma escriturística en particular de las construcciones de las explicaciones de los acontecimientos, referido a la construcción narrativa de procesos causales, lo cual permite una forma específica de pensar y representar la realidad, a partir de la comprensión crítica del pasado. (PLÁ, 2005). Por tanto, el pensamiento histórico resulta un modo más de aprehensión de la realidad, si se trata de insistir en las ventajas de pensar históricamente en el proceso de enseñanza-aprendizaje de la historia en la escuela. (PULIDO, 2009, p. 235).

Junto a lo anterior, un elemento del marco curricular que debemos considerar es la idea de una construcción gradual, lo cual favorece el desarrollo significativo del pensamiento histórico, pues, este constituye una herramienta necesaria para que los estudiantes puedan gestionar una visión crítica y comprensiva tanto de su entorno como del mundo de manera creciente. De este modo, la enseñanza de la asignatura sitúa al pensamiento histórico como una herramienta para el desarrollo del razonamiento crítico, razón por la cual se insiste en que dicha habilidad permite que los estudiantes puedan pensar su propia historia. En este aspecto, se propone el planteamiento de situaciones de aprendizaje que inviten a los estudiantes a preguntarse sobre el pasado, a conocer distintas visiones e interpretaciones sobre los acontecimientos y a buscar explicaciones que le permitan reconocer el sentido de los hechos del pasado y del presente (MINEDUC, 2013, p. 194), considerando que el pensamiento histórico es herramienta que permite crear vínculos de pertenencia con la sociedad y, por tanto, favorece la comprensión de la realidad.

Asimismo, se explicita que una parte fundamental en el tratamiento de esta habilidad consiste en que los estudiantes reconozcan las relaciones dinámicas de continuidad y cambio a través del tiempo, los múltiples antecedentes y causas que explican el devenir de la sociedad y que comprendan que la historia es una construcción humana. En consecuencia, se espera que los estudiantes sean capaces de comprender las múltiples formas en que el pasado marca el acontecer del mundo contemporáneo, poniéndolo en perspectiva y evidenciando el carácter contingente del mismo. (MINEDUC, 2013, p. 195).

\section{El desarrollo de habilidades cognitivas}

Los objetivos de aprendizaje de historia, geografía y ciencias sociales contemplan una serie de habilidades propias de las disciplinas que conforman 
estas asignaturas. Estas destrezas son valiosas herramientas cognitivas necesarias para comprender los contenidos estudiados y para adquirir conocimientos en otras áreas y en diferentes contextos de la vida. Por lo anterior, se espera que la adquisición de conocimientos y el desarrollo de la capacidad de procesar la información contribuyan a que los alumnos puedan tomar decisiones de una manera responsable e informada en el contexto de la vida democrática y la participación responsable en ella. (MINEDUC, 2013, p. 200).

De esta forma, en cuanto a las habilidades que el currículo escolar chileno propone para la educación media se plantean los mismos desempeños del ciclo anterior de educación básica (primaria). No obstante a ella, presentan mayor complejidad cognitiva ya que se concibe el aprendizaje de la historia, la geografía y las ciencias sociales como un proceso progresivo y continuo de desarrollo de contenidos conceptuales, procedimentales y actitudinales. Estas habilidades están referidas a:

- Pensamiento temporal y espacial;

- Análisis y trabajo con fuentes;

- Pensamiento crítico;

- Habilidades de comunicación.

El pensamiento temporal y espacial se plantea para esta etapa como la continuidad de la aprehensión temporal y la aplicación de los conceptos de tiempo y espacio, proceso iniciado en el ciclo anterior, con la finalidad de que los alumnos puedan orientarse, contextualizar, ubicar y comprender los procesos y acontecimientos estudiados así como también relacionar los sucesos con su propia realidad. Por dicho motivo, en estos niveles se considera que los estudiantes aprenderán a establecer y representar secuencias cronológicas entre periodos históricos y a comparar procesos históricos, identificando relaciones de causalidad, continuidades y cambios. (MINEDUC, 2013, p. 201). La finalidad más inmediata de esta habilidad es que los estudiantes sean capaces de identificar elementos comunes en el pasado que constituyen los fenómenos históricos, donde la ubicación espacial requeriría el uso de recursos geográficos que permitan al alumno observar patrones y asociaciones en el entorno, para permitir así obtener la comprensión de la dimensión espacial de los fenómenos estudiados.

Considerando que los conceptos relacionados con la temporalidad histórica se plantean como una habilidad que debe desarrollarse paulatinamente, se espera que los alumnos desarrollen la habilidad de comprender para aplicar conceptos más complejos del tiempo histórico, por ejemplo fortaleciendo el reconocimiento de los distintos ritmos y duraciones de los procesos de la historia. (MINEDUC, 2013, p. 196). 
En cuanto al análisis y trabajo con fuentes de información, se busca fortalecer el desarrollo de desempeños cognitivos relacionado con la indagación y, junto a ello, trabajar en la rigurosidad científica, la estructuración clara y ordenada de ideas, la perseverancia, el trabajo colaborativo y el interés por conocer temas nuevos para avanzar a distintas áreas del conocimiento. Aspectos que nos parecen que estarían vinculados no sólo a habilidades procedimentales sino que también a contenidos actitudinales propicios para el aprendizaje. Así, los estudiantes deben ser capaces de realizar proyectos de investigación, elaborar hipótesis, así como aplicar distintas estrategias para registrar, citar y organizar la información obtenida (MINEDUC, 2013, p. 201), muchos de estos elementos se consideran centrales dentro de la metodología histórica, por ello el análisis y contrastación de distinta evidencia le permitirá al estudiante obtener información relevante, formularse preguntas, establecer relaciones, elaborar conclusiones y resolver problemas. Por esta razón, se pretende que el alumno avance sobre la evaluación de las fuentes históricas como fuentes de información, como un ámbito central de las actividades de exploración, introduciendo al estudiante en la lógica científica de la historia (FELIÚ; HERNÁNDEZ, 2011, p. 55), tal como ya hemos enfatizado antes, porque facilitarían una visión más cercana de los procesos históricos. En este sentido, puede mostrarles cómo los historiadores crean versiones equilibradas buscando fuentes con diversos grados de tendenciosidad hacia uno u otro lado. Puede hacer una demostración práctica de ese tipo de ejercicio proporcionando a sus alumnos dos fuentes sesgadas o contrapuestas y pidiéndoles que extraigan de ellas los hechos que más probablemente fueron verdad a partir del descubrimiento de elementos mencionados por ambas fuentes. (MURPHY, 2011, p. 101).

Respecto al pensamiento crítico, para este ciclo se promueve la capacidad de integrar elementos para elaborar procesos analíticos y, junto a ello, reconocer la complejidad inherente a los fenómenos humanos que estudian la historia y las ciencias sociales. En lo particular, y en relación a lo expuesto anteriormente, se busca reforzar el interés por conocer y evaluar las distintas visiones e interpretaciones que existen sobre los fenómenos estudiados (MINEDUC, 2013, p. 202), lo que podemos relacionar con actividades de contrastación de evidencia histórica diversas. Y de esta manera, comprender que las sociedades se construyen histórica y geográficamente, es decir, que están contextualizadas o responden a un tiempo y en un espacio determinado considerando que han sido moldeadas por diferentes influencias así como que existen relaciones de cambio y continuidad en los procesos que la estructuran.

Por tanto, el pensamiento crítico busca favorecer la explicación de los procesos históricos, enfocándose simultáneamente en habilidades propias del pensamiento histórico, permitiendo que los estudiantes reconozcan el nivel in- 
terpretativo del quehacer de la historia y de las ciencias sociales, debido a que mediante la evaluación de los argumentos y de la evidencia que sustentan cada perspectiva, el estudiante desarrolla su propia representación respecto de los procesos históricos. De hecho, la comprensión que los sujetos tienen acerca de la historia dependen del método empleado (CARRETERO; VOSS, 2004, p. 326), porque estas habilidades son de análisis crítico y de pensamiento superior, que permiten que los estudiantes adquieran una rol activo dentro de la construcción de su conocimiento, situación didáctica que estaría en la línea de la perspectiva constructivista del aprendizaje.

Para el caso de la habilidad de comunicación, no se presentan cambios evidentes según lo planteado en el ciclo $1^{\circ}$ a $6^{\circ}$ básico, donde se proponía el desarrollo de la comunicación oral y escrita expresado a través de investigaciones, utilizando una estructura clara, completa y efectiva. Así, los desempeños asociados a la comunicación buscan reforzar en los estudiantes la capacidad de transmitir los resultados de sus indagaciones de forma clara y respetuosa, por medio de diferentes formas de expresión. (MINEDUC, 2013, p. 184). Por tanto, se busca desarrollar también la capacidad de hablar y escribir de forma correcta, así como promover el diálogo y respetar opiniones diferentes a las propias, a partir de actividades como debates, juicios históricos o creaciones de textos argumentativos.

\section{Los contenidos actitudinales}

Las bases curriculares de historia, geografía y ciencias sociales promueven un conjunto de actitudes para todo el ciclo de formación general, que derivan de los Objetivos de Aprendizaje Transversales (OAT) y se orientan al desarrollo personal y social de los estudiantes. Dada su relevancia en el aprendizaje en el contexto de cada disciplina, estas se deben desarrollar de manera integrada con los conocimientos y las habilidades propios de la asignatura (MINEDUC, 2013, p. 202), se plantean acompañadas de los valores que se desean desarrollar como manifestaciones permanentes del aprendizaje. Según plantea el currículo, las actitudes deben ser promovidas para la formación integral de los estudiantes y son de especial relevancia para el aprendizaje y, por ello, se orientan a desarrollar de manera integrada los conocimientos y habilidades propios de la disciplina. De esta forma, las actitudes han sido diseñadas para su desarrollo progresivo, ya que se presentan transversalmente a la enseñanza de la historia desde $1^{\circ}$ básico a $4^{\circ}$ medio, incluyendo los electivos humanistas de $3^{\circ}$ y $4^{\circ}$ medio. 
Las actitudes a desarrollar en la asignatura hacen referencia, principalmente, a aspectos de la formación democrática de la ciudadanía tales como igualdad de derechos, respeto a la diversidad, valoración democrática, identidad cultural, responsabilidad social, desarrollo sustentable, entre otros. Y, en menor medida, pero no por ello menos relevante, a elementos metodológicos y/cognitivos tales como el aporte de las ciencias sociales, desarrollo del pensamiento autónomo, capacidad reflexiva y uso de nuevas tecnologías, por nombrar algunas. De esta forma, las actitudes a desarrollar en la asignatura de historia, geografía y ciencias sociales son:

- Respetar y defender la igualdad de derechos esenciales de todas las personas, sin distinción de raza o etnia, nacionalidad, situación socioeconómica, idioma, ideología u opinión política, religión o creencia, sindicación o participación en organizaciones gremiales o la falta de ellas, género, orientación sexual, estado civil, edad, filiación, apariencia personal, enfermedad o discapacidad;

- Respetar la diversidad cultural, religiosa y étnica, y las ideas y creencias distintas de las propias, considerando la importancia del diálogo para la convivencia y el logro de acuerdos, evitando el conocimiento histórico como una forma de prejuicios;

- Pensar en forma autónoma y reflexiva, fundamentar las ideas y posturas propias, y desarrollar una disposición positiva a la crítica y la autocrítica;

- Demostrar valoración por el aporte de las ciencias sociales a la comprensión de la realidad humana y su complejidad, mediante distintas herramientas metodológicas y perspectivas de análisis;

- Demostrar valoración por la democracia, reconociendo la importancia de ser ciudadanos activos, solidarios y responsables, conscientes y comprometidos con el ejercicio de sus derechos y deberes;

- Demostrar valoración por la vida en sociedad, a través del compromiso activo con la convivencia pacífica, el bien común, la igualdad de hombres y mujeres y el respeto a los derechos fundamentales de todas las personas;

- Demostrar interés por conocer el pasado de la humanidad y el de su propia cultura, y valorar pertenencia con la sociedad en sus múltiples dimensiones (familia, localidad, región, país, humanidad, etc.);

- Desarrollar actitudes favorables a la protección del medio ambiente, demostrando conciencia de su importancia para la vida en el planeta y una actitud propositiva ante la necesidad de lograr un desarrollo sustentable;

- Demostrar una actitud propositiva para contribuir al desarrollo de la sociedad, mediante iniciativas que reflejen responsabilidad social y 
creatividad en la búsqueda de soluciones, perseverancia, empatía y compromiso ético con el bien común;

- Usar de manera responsable y efectiva las tecnologías de la comunicación para la obtención de información y la elaboración de evidencia, dando crédito al trabajo de otros y respetando la propiedad y la privacidad de las personas.

\section{A modo de conclusión}

Los avances de la historia como saber científico han significado un cambio en las metodologías de estudio y las fuentes de información que permiten construir el conocimiento histórico, lo cual ha significado pasar desde un relato lineal de hechos y consecuencias a una explicación multicausal de procesos estructurales que han dado sentido al pasado.

Así, el aprender a enseñar debe estar vinculado estrechamente al enseñar a pensar históricamente, relacionado con la comprensión del conocimiento histórico a partir de la comprensión crítica del pasado. Por eso que algunos autores no han dudado en afirmar que los maestros de historia deben ir más allá de repetir una serie de contenidos o pensar históricamente para sí mismos, sino que deben ayudar a sus alumnos a aprender historia junto con aprender a pensar históricamente (BAIN, 2005, p. 2), por eso proponen transformar los contenidos curriculares en problemas historiográficos que comprometan al estudiante con el pensamiento de la historia, en vista de que, según el investigador, rara vez los objetivos curriculares conectan los resultados con sus raíces intelectuales, con los problemas e interrogantes que inicialmente dieron lugar a tal entendimiento, de modo que dichos objetivos no estarían proporcionando las conexiones, patrones o relaciones disciplinares que permiten a los docentes y a los alumnos construir cuadros coherentes de la historia que estudian. Para buscar la solución a este conflicto, el autor sugiere que la organización del currículo deba realizarse en torno a conceptos claves de la historia, grandes ideas e interrogantes centrales, ya que cuando a los profesores se les exige que se limiten a seguir los libros de texto, o a enseñar de acuerdo con programas basados en la competencia específica, sienten que las pautas curriculares reducen su capacidad para adaptar la enseñanza a sus estudiantes. (DARLING, 2001, p. 9).

Los elementos anteriores son importantes de considerar porque la enseñanza de la historia sigue creando problemas ente quienes la enseñan y quienes la aprenden (FUNES, 2006, p. 257) y cada cierto tiempo se presenta el problema de 
que la escuela no ha logrado desarrollar y extender una pedagogía generalizada para la comprensión, de forma tal que los estudiantes gocen de oportunidades para evaluar y aplicar sus ideas, contemplar los conocimientos desde diversos puntos de vista o alcanzar niveles de logro escolar efectivos. La situación anterior influye directamente en la transmisión de la historia que se ha realizado al interior del aula de clases y, por ello, el desarrollo del pensamiento histórico en los estudiantes escolares ha sido una tarea compleja que ha debido enfrentar el profesor de la escuela cuando afronta la enseñanza del contenido histórico.

Por tanto, los profesores en formación deben tener una compresión acabada del pensamiento histórico, porque si su concepción se presenta de forma tradicional, enfocada sólo en procesos unicausales, en la sala de clase desarrollarán una visión parcelada del conocimiento de la historia, diferenciándose de lo central de un proceso de aprendizaje significativo que es potenciar las habilidades de indagación y análisis histórico.

\section{REFERENCIAS}

ARRANZ, L. La historia y su vocación integradora e interdisciplinar. En: GARCÍA, M. Un curriculum de ciencias sociales para el siglo XXI: qué contenidos y para qué enseñar. Logroño: Diada, 1999.

BAIN, R. Cómo aprenden los estudiantes de historia en el aula de clases. “¿Ellos pensaban que la tierra era plana?" Aplicación de los principios de Cómo aprende la gente, en la enseñanza de la Historia en la Educación Secundaria. En: DONOVAN, S.; BRANSFORD, J. How Students Learn: History, Mathematics, and Science in the Classroom. Washington D.C.: Editorial de la Academia Nacional de Ciencias de Estados Unidos, 2005. Disponible en: <http://www.eduteka.org/ComoAprendenLosEstudiantes.php>. Acceso en: 25 abr. 2015.

CARRETERO, M.; VOSS, J. F. (Comps.). Aprender y pensar historia. Buenos Aires: Amorrortu, 2004.

DARLING, L. El derecho de aprender. Buenas escuelas para todos. Barcelona: Editorial Ariel, 2001

EGGEN, P. Estrategias docentes. Enseñanza de contenidos curriculares y desarrollo de habilidades. México: Fondo de Cultura Económica, 2001.

FELIÚ, M.; HERNÁNDEZ, X. 12 ideas clave. Enseñar y aprender historia. Barcelona: Editorial GRAÓ, 2011. 
FUNES, A. La enseñanza de la historia y sus significaciones. En: GOMEZ, A.; NUÑEZ, M. Formar para investigar. Investigar para formar en didáctica de las ciencias sociales. Antequera: Asociación Universitaria del Profesorado de Didáctica de las Ciencias Sociales, 2006.

LÓPEZ FACAL, R. La LOMCE y la competencia histórica. Revista Ayer, Madrid, v. 2, n. 9 , p. $273-285,2014$.

MINEDUC. Currículum de la educación media. Objetivos fundamentales y contenidos mínimos obligatorios. Santiago: MINEDUC, 2005.

MINEDUC. Marco Curricular historia, geografia y ciencias sociales. Santiago de Chile: MINEDUC, 2009.

MINEDUC. Bases curriculares historia, geografía y ciencias sociales. $1^{\circ}$ a $6^{\circ}$ básico. Santiago de Chile: MINEDUC, 2012.

MINEDUC. Bases curriculares historia, geografia y ciencias sociales. $7^{\circ}$ básico a $2^{\circ}$ medio. Santiago de Chile: MINEDUC, 2013.

MURPHY, J. Más de 100 ideas para enseñar historia. Primaria y secundaria. Barcelona: Editorial GRAÓ, 2011.

PLÁ, S. Aprender a pensar historicamente. La escritura de la historia en el bachillerato. México D.F: Colegio Madrid/Plaza y Valdés, 2005.

PRATS, J. Enseñanza y aprendizaje de la historia en la educación básica. México D.F.: Secretaria de Educación, 2001. (Teoría y práctica curricular de la educación básica).

PULIDO, M. Pensamiento complejo: una perspectiva para enseñar a pensar la historia en el contexto del aula. Ciencia y sociedad, v. XXXIV, n. 2, p. 234-263, abr./jun. 2009.

VÁSQUEZ, N.; MONTECINOS, C. Las competencias específicas de los profesores de historia y ciencias sociales. IBER. Revista de Didáctica de las Ciencias Sociales, Geografía e Historia, Barcelona, v. 1, n. 52, p. 18-29, 2007.

Texto recibido en 22 de marzo de 2016. Texto aprobado en 27 de marzo de 2016. 